\title{
An FHL1-containing complex within the cardiomyocyte sarcomere mediates hypertrophic biomechanical stress responses in mice
}

\author{
Farah Sheikh, ${ }^{1}$ Anna Raskin,, ${ }^{1,2}$ Pao-Hsien Chu, ${ }^{3}$ Stephan Lange, ${ }^{1}$ Andrea A. Domenighetti, ${ }^{1}$ \\ Ming Zheng, ${ }^{1}$ Xingqun Liang, ${ }^{1}$ Tong Zhang, ${ }^{4}$ Toshitaka Yajima, ${ }^{1}$ Yusu Gu, ${ }^{1}$ Nancy D. Dalton, ${ }^{1}$ \\ Sushil K. Mahata, ${ }^{1,5}$ Gerald W. Dorn II,6 Joan Heller-Brown, ${ }^{4}$ Kirk L. Peterson, ${ }^{1}$ \\ Jeffrey H. Omens, ${ }^{1,2}$ Andrew D. McCulloch, ${ }^{2}$ and Ju Chen ${ }^{1}$ \\ 1Department of Medicine and 2Department of Bioengineering, UCSD, La Jolla, California, USA \\ ${ }^{3}$ Department of Internal Medicine, Chang Gung Memorial Hospital, Chang Gung University College of Medicine, Taipei, Republic of China. \\ 4Department of Pharmacology, UCSD, La Jolla, California, USA. 5VA San Diego Healthcare System, San Diego, California, USA. \\ ${ }^{6}$ Department of Medicine and Center for Pharmacogenomics, Washington University School of Medicine, St. Louis, Missouri, USA.
}

\begin{abstract}
The response of cardiomyocytes to biomechanical stress can determine the pathophysiology of hypertrophic cardiac disease, and targeting the pathways regulating these responses is a therapeutic goal. However, little is known about how biomechanical stress is sensed by the cardiomyocyte sarcomere to transduce intracellular hypertrophic signals or how the dysfunction of these pathways may lead to disease. Here, we found that fourand-a-half LIM domains 1 (FHL1) is part of a complex within the cardiomyocyte sarcomere that senses the biomechanical stress-induced responses important for cardiac hypertrophy. Mice lacking Fbl1 displayed a blunted hypertrophic response and a beneficial functional response to pressure overload induced by transverse aortic constriction. A link to the $\mathrm{G} \alpha \mathrm{q}(\mathrm{Gq})$ signaling pathway was also observed, as Fbl1 deficiency prevented the cardiomyopathy observed in Gq transgenic mice. Mechanistic studies demonstrated that FHL1 plays an important role in the mechanism of pathological hypertrophy by sensing biomechanical stress responses via the N2B stretch sensor domain of titin and initiating changes in the titin- and MAPK-mediated responses important for sarcomere extensibility and intracellular signaling. These studies shed light on the physiological regulation of the sarcomere in response to hypertrophic stress.
\end{abstract}

\section{Introduction}

Cardiovascular disease and ensuing heart failure is the leading cause of human morbidity and mortality worldwide. Biomechanical stress-induced growth responses have been shown to play a pivotal role in the pathophysiology of cardiac disease and could have application for therapies. However, little is known about how biomechanical stress is sensed by the cardiomyocyte sarcomere and transduced into intracellular signals or how dysfunction of these pathways may lead to disease. Human genetic studies and genetic mouse models have revealed a structural role for cytoskeletal proteins in the pathophysiology of cardiac disease (1-8). However, evidence suggests that these proteins may also function outside their traditional roles in the cardiac cytoskeleton and have mechanotransduction functions in response to biomechanical stress $(4,5,9,10)$. A central challenge is to define the precise molecular signals that link these cytoskeletal proteins and their functions to disease in vivo.

Nonstandard abbreviations used: $+\mathrm{dP} / \mathrm{dt}$ maximum, maximum positive first derivative of $\mathrm{LV}$ pressure as measured over time; $-\mathrm{dP} / \mathrm{dt}$ maximum, maximum negative first derivative of LV pressure as measured over time; FHL1, four-and-a-half LIM domains 1; Gq, Gaq; Gq+, Gq gain-of-function transgenic (mice); IVSd, interventricular septal wall thickness; $\mathrm{KO} / \mathrm{Gq}, F b l 1^{-/} / \mathrm{Gq}$ transgenic; $L_{\max }$, muscle length at which the muscle develops the maximum force; LV FS, percentage of LV fractional shortening; LVPWd, LV posterior wall thickness; TAC, transverse aortic constriction.

Conflict of interest: The authors have declared that no conflict of interest exists. Citation for this article: J. Clin. Invest. doi:10.1172/JCI34472.
Cytoskeletal proteins from the LIM domain family have been thought to play a pivotal role in biomechanical stress responses leading to muscle disease $(4,8)$. The four-and-a-half LIM domains (FHL) family is a newly identified group of LIM-only proteins named and structurally characterized by 4 complete LIM domains and an N-terminal half LIM domain (11). FHL1, -2 , and -3 are enriched in mammalian striated muscle and associated with multiple binding partners and biological functions $(12,13)$, including striated muscle development and function (14). However, the physiological relevance of these multiple interactions and functions of FHL proteins in vivo remain unclear. A growing body of evidence has suggested that FHL1 may play an important role in biomechanical stress responses involved in cardiac hypertrophy and disease. Recent studies have shown FHL1 as the only FHL member to be upregulated in mouse hearts subsequent to in vivo pressure overload induced hypertrophy and hypertrophic agonists $(14,15)$. These results are consistent with observations in humans, which show that FHL1 is the only FHL member that is dramatically increased in hearts of human patients exhibiting hypertrophic cardiomyopathy (16-18). Based on these findings, we hypothesized that FHL1 would play an essential role in biomechanical stress-induced cardiac hypertrophy and disease in vivo.

In the present study, we uncovered the role of FHL in the myocardium in vivo by generating and characterizing Fbl1-deficient $\left(F_{b l 1^{-/}}\right)$ mice. Our studies demonstrate a critical role for FHL1 to sense biomechanical stress responses important in pathological cardiac hypertrophy. Using a combination of in vitro and in vivo systems, we 
demonstrate interactions of FHL1 with both sarcomeric (titin N2B) and signaling (Goq-MAPK [Gq-MAPK] cascade) components within the cardiomyocyte and demonstrate the ability of these interactions to dually affect sarcomere extensibility and hypertrophic signaling responses. Taken together, our studies demonstrate that FHL1 provides an essential connection between the muscle stretch sensor and downstream titin- and MAPK-mediated responses important in biomechanical stress responses involved in cardiac hypertrophy. These studies further shed light on the physiological regulation of the sarcomere in response to hypertrophic stress.

\section{Results}

Generation of Fbl1 $1^{-/-}$mice. To examine the role of FHL1 in the myocardium in vivo, we generated $F b l 1^{--}$mice (Figure 1, A and B). Western blot analysis demonstrated loss of FHL1 but no change in FHL2 protein expression in adult striated muscle of $\mathrm{Fbl1}^{-/-}$mice (Figure 1C). Fbl1-/- mice were born at the expected Mendelian ratios and were viable, with normal life spans. Fbl1 ${ }^{-/-}$hearts exhibited no differences in cardiac size, dimensions, and function from 8 weeks (Table 1) to 1 year of age (data not shown) when compared with WT mice. In addition, no significant differences in blood pressure were observed between WT and $\mathrm{Fbl1}^{-/-}$mice (Supplemental Table 1; supplemental material available online with this article; doi:10.1172/JCI34472DS1).

Fbl1 ${ }^{-1}$ mice exhibit a blunted and beneficial response to pressure overload induced by transverse aortic constriction. Studies have implicated a role for FHL1 in stress-induced cardiac hypertrophy (14, 16-18). As a result, we tested the consequences of Fbl1 deficiency in an in vivo model of pressure overload-induced cardiac hypertrophy caused by transverse aortic constriction (TAC). Similar trans-stenotic pressure gradients were observed in WT and $\mathrm{Fhl1}^{-/-}$mice following TAC (Table 1). However, $\mathrm{Fbl1}^{-/}$hearts were significantly smaller than those of controls at 1 and 5 weeks after TAC (Figure 1D). This was reflected in the significantly smaller increase in LV/BW ratio (Figure $1 \mathrm{E}$ ), cardiomyocyte cross-sectional area (Figure $1 \mathrm{~F}$ ), LV posterior wall thickness (LVPWd), and interventricular septal wall thickness (IVSd) (Table 1) in Fbl1 - $^{--}$hearts compared with those of controls. A blunted response to hypertrophy was further indicated by the significantly smaller increase in expression of the fetal gene markers ANF, $\beta$-MHC, and skeletal $\alpha$-actin, known to be re-expressed following TAC, in $\mathrm{Fbl1}^{-/-}$ventricles compared with those of controls (Figure 1G). Remarkably, echocardiography demonstrated that Fbl1 ${ }^{-1}$ mice subjected to TAC were able to maintain the percentage of LV fractional shortening (LV FS) at levels comparable with those of sham-operated controls (Table 1), demonstrating preservation of function in the context of pressure overload. In contrast, controls exhibited early signs of heart failure, including a significant decrease in LV FS and increased chamber dilation (increased LV internal dimension at end diastole [LVIDd] and LV internal dimension at end-systole), which were clearly apparent 5 weeks after TAC (Table 1). Hemodynamic assessment of Fbl1 ${ }^{-/-}$hearts after long term TAC revealed a significant preservation in $\mathrm{LV}$ end-diastolic pressure and systolic function as assessed by maximum positive first derivative of $\mathrm{LV}$ pressure as measured over time ( $+\mathrm{dP} / \mathrm{dt}$ maximum), as well as increased diastolic function as assessed by maximum negative first derivative of $L V$ pressure as measured over time (- $\mathrm{dP} / \mathrm{dt}$ maximum) and relaxation time constant when compared to control mice after TAC and SHAM controls (Supplemental Table 2).

Genetic ablation of Fhl1 is sufficient to prevent cardiomyopathy in the Gq transgenic model. Gq signaling plays an essential role in cardiac hypertrophy induced by agonists in vitro and TAC in vivo (19-21). To explore possible links between FHL1 and Gq signaling, we first examined the possibilities that GPCR signaling could regulate endogenous FHL1 expression in cardiomyocytes. GPCR agonists, phenylephrine, and angiotensin II, as well as constitutively active Gq overexpression, significantly increased FHL1 expression in postnatal cardiomyocytes in vitro and in mouse hearts in vivo (Figure 2A). Fbl1 $1^{-/-}$mice were bred with the cardiomyopathic Gq transgenic mice to determine the consequences of Fhl1 deficiency on Gq signaling in the postnatal heart. Fbl1 deficiency completely prevented the cardiomyopathy in the $\mathrm{Gq}$ transgenic mice. Compared with $\mathrm{Gq}$ transgenic ventricles, double Fbl1-/-/Gq transgenic (KO/Gq) hearts exhibited a significant reduction in LV size, as assessed by LV/BW ratios and histological assessment of heart size (Figure 2B), as well as expression of fetal gene markers known to be upregulated with hypertrophy and stress (Figure 2C). Chamber size (LVIDd), wall thicknesses (LVPWd, IVSd), and function (LV FS) in KO/Gq transgenic hearts were comparable with WT and $\mathrm{Fbl1}^{-/}$hearts (Figure 2D). This is in contrast with Gq hearts, which exhibited a significant increase in LVIDd, LVPWd, and IVSd as well as a decrease in LV FS (Figure 2, B and D), consistent with previous findings (22).

FHL1 associates with MAPK cascade components at the sarcomeric I band. A recent study has demonstrated that FHL proteins physically associate with the MAPK component, ERK2 (23). Since LIM proteins can act as scaffolding proteins (13), we sought to determine whether FHL1 could associate with all or certain MAPK components. Direct interactions between FHL1 and MAPK components were investigated by yeast 2 -hybrid, and interactions were quantified by $\beta$-galactosidase activities (Table 2). Specific interactions between full-length FHL1 (4.5 LIM) and Raf1, MEK2, and ERK2, but not MEK1, were observed, suggesting that FHL1 can interact with inactive forms of Raf/MEK/ERK. A specific interaction between FHL1 and a constitutively phosphorylated mutant of ERK2 (i.e., ERK2 [TYDD]) was also observed, suggesting that FHL1 can interact with active forms of ERK2. Deletion analysis revealed that the first 2 full LIM domains (FHL1 2 LIM) are essential for the interaction between FHL1 and Raf1, MEK2, ERK2, and ERK2 (TYDD). In addition, no interactions could be observed between the small isoform of FHL1 (KyoT2), which is not expressed in the heart (data not shown), and Raf1, MEK2, ERK2, and ERK2 (TYDD). A link between FHL1 and the Gq-MAPK was also shown when the MAPK/ERK1 inhibitor, PD98059, could completely block phenylephrine- and angiotensin II-mediated upregulation of FHL1 expression in cardiomyocytes (our unpublished observations).

Coimmunoprecipitation assays were performed on WT mouse hearts at basal conditions and following TAC to assess the endogenous association of Raf1, MEK1/2, and ERK2 with FHL1. Specific interactions were demonstrated between endogenous FHL1 and Raf1, MEK1/2, and ERK2 in adult mouse hearts, which were increased following TAC (Figure 3A), consistent with yeast 2-hybrid studies (Table 1). Immunofluorescence microscopy was further utilized to assess the localization of FHL1 and Raf/MEK/ERK in adult cardiomyocytes. Using FHL1 antibodies, which were verified in $\mathrm{Fbl1}^{-/-}$mice (Figure 1C), as well as sarcomeric antibodies for the I band (titin N2B) we demonstrated the localization of FHL1 as a narrow doublet directly colocalizing with titin N2B in adult cardiomyocytes (Figure 3B). To determine whether FHL1 directly binds to titin N2B, a minimal element of titin N2B (GFP-htitinN2B us3) that has also been previously shown to bind to FHL2 (24) was generated and assayed for its ability to interact with FHL1 in COS cells. 
A FHL1 gene loc
Targeting cons
FHL1 mutant
gene locus

D Sham WT

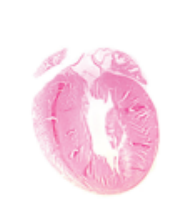

$\mathrm{KO}$

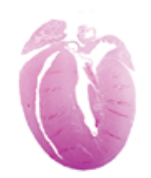

F

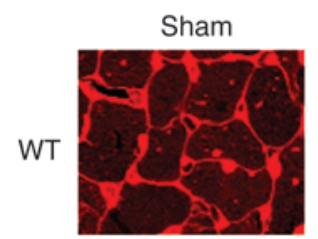

KO

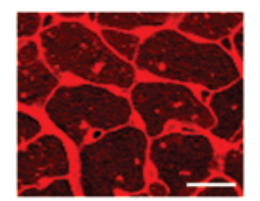

G

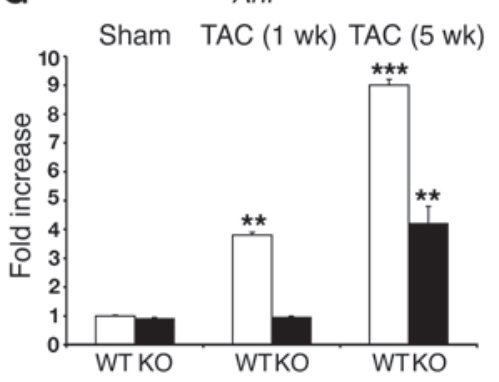

$1 \mathrm{~kb}$
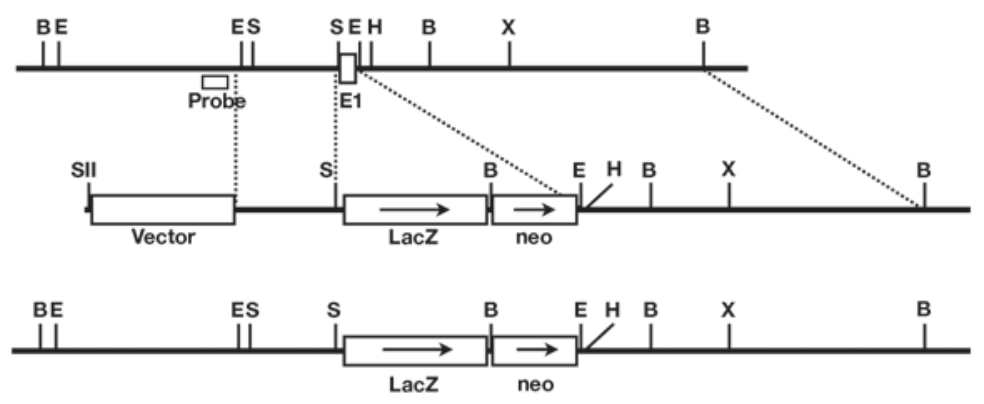

B

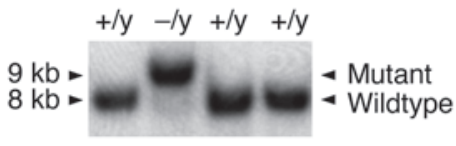

C WT KO

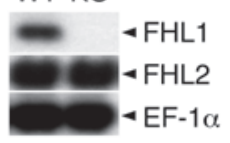

E
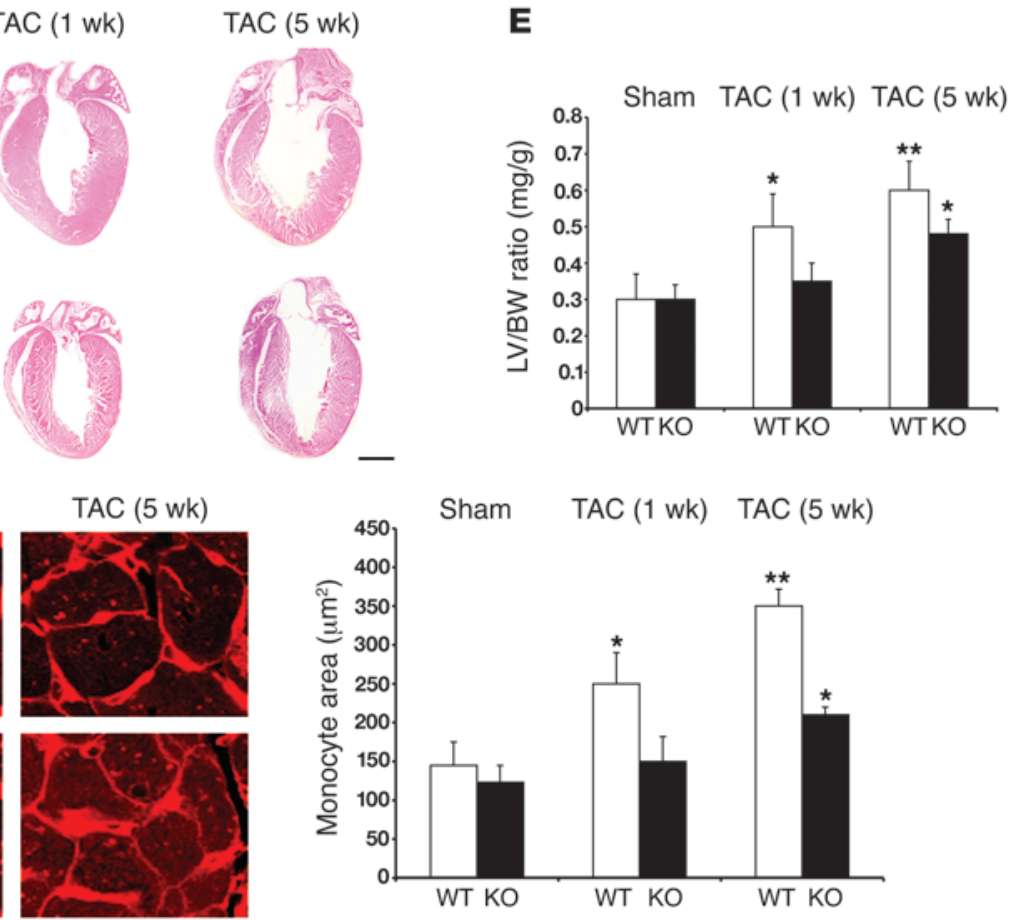

$\beta-M H C$
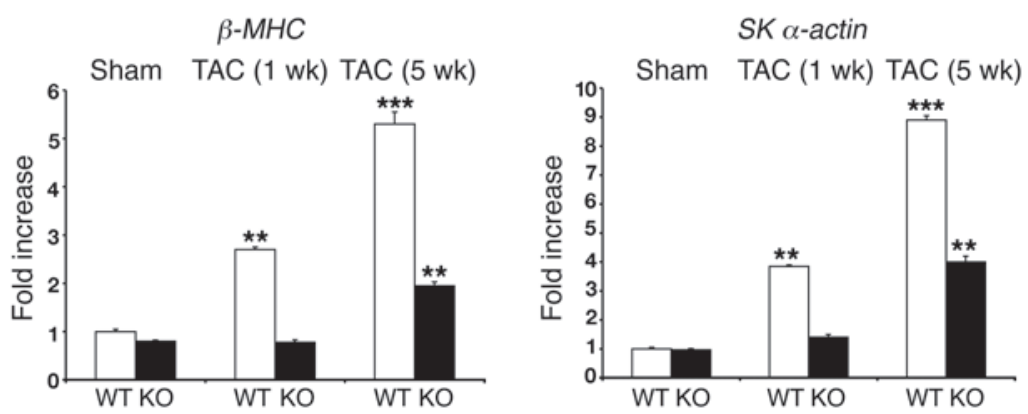

\section{Figure 1}

Targeted deletion of Fhl1 in mice and its effect on in vivo pressure overload-induced cardiac hypertrophy. (A) Fh/1 genomic region of interest (top), the targeting construct (center), and the mutated locus after homologous recombination (bottom). B, BamHI; E, EcoRl; E1, exon 1; $\mathrm{H}$, HindIII; N, Notl; S, Stul; SII, SstIl; and X, Xhol. (B) Neo-positive ES cell DNAs were digested with BamHI and analyzed by Southern blotting for WT (+/y) and targeted (-/y) alleles with the probe shown in A. (C) Protein $(20 \mu \mathrm{g})$ from WT and Fh/1-deficient (KO) ventricles (8 weeks old) were subjected to SDS-PAGE to detect FHL1 $(\sim 32 \mathrm{kDa})$ and FHL2 $(\sim 34 \mathrm{kDa})$ expression. EF-1 $\alpha(\sim 53 \mathrm{kDa})$ protein was used to normalize for loading. (D) Paraffin sections from WT and KO hearts following sham (1 week) and TAC operation were stained with hematoxylin and eosin. Scale bar: $2.5 \mathrm{~mm}$. (E) LV weight/BW ratios in WT and KO mice following sham (1 week) and TAC operation. (F) Ventricular sections from WT and KO mice, following sham (1 week) and TAC operation, stained with wheat germ agglutinin and Hoechst 33342. Original magnification, $\times 400$. Myocyte cross-sectional areas from at least 200 myocytes per heart in randomly selected fields were assessed. Scale bar: $16 \mu \mathrm{m}$. (G) Anf, $\beta$-MHC, and skeletal $\alpha$-actin RNA expression in WT and KO LVs $(n=4)$ following sham (1 week) and TAC operation were normalized to Gapdh expression and presented as fold induction relative to WT sham controls. ${ }^{*} P<0.05 ;{ }^{* \star} P<0.01 ;{ }^{* \star *} P<0.001$. 


\section{Table 1}

In vivo echocardiographic assessment of cardiac size and function in WT and Fh/1-/- mice following TAC at 1 and 5 weeks

\begin{tabular}{|c|c|c|c|c|c|c|c|c|}
\hline & Sham, 1 wk & \multicolumn{3}{|c|}{ TAC, 1 wk } & \multirow{2}{*}{$\begin{array}{l}\text { Sham, } 5 \text { wk } \\
\text { WT }(n=12)\end{array}$} & \multicolumn{3}{|c|}{ TAC, 5 wk } \\
\hline & WT $(n=11)$ & Fhl1-/ $(n=10)$ & WT $(n=12)$ & Fhl1-/- $(n=10)$ & & Fhl1-/- $(n=10)$ & WT $(n=13)$ & Fhl1-/- $(n=10)$ \\
\hline BW, g & $27.1 \pm 3.4$ & $24.9 \pm 3.8$ & $27.8 \pm 4.32$ & $25.8 \pm 3.0$ & $28.3 \pm 1.7$ & $27.6 \pm 1.7$ & $31.8 \pm 1.8$ & $32 \pm 1.8$ \\
\hline $\mathrm{HR}, \mathrm{bpm}$ & $511 \pm 64$ & $552 \pm 58$ & $498 \pm 43$ & $521 \pm 76$ & $572 \pm 46$ & $563 \pm 50$ & $607 \pm 30$ & $605 \pm 45$ \\
\hline IVSd, mm & $0.75 \pm 0.08$ & $0.71 \pm 0.07$ & $0.9 \pm 0.09^{A}$ & $0.73 \pm 0.06$ & $0.61 \pm 0.05$ & $0.6 \pm 0.08$ & $0.8 \pm 0.05^{A}$ & $0.7 \pm 0.05$ \\
\hline LVIDd, mm & $3.68 \pm 0.44$ & $3.36 \pm 0.27$ & $3.62 \pm 0.41$ & $3.43 \pm 0.36$ & $3.8 \pm 0.03$ & $3.7 \pm 0.16$ & $4.2 \pm 0.04^{A}$ & $3.95 \pm 0.03$ \\
\hline LVIDs, mm & $0.84 \pm 0.22$ & $0.78 \pm 0.16$ & $1.75 \pm 0.70$ & $1.33 \pm 0.50$ & $2.1 \pm 0.1$ & $1.89 \pm 0.60$ & $2.8 \pm 0.3^{A}$ & $2.26 \pm 0.20$ \\
\hline LVPWd, mm & $0.75 \pm 0.07$ & $0.70 \pm 0.07$ & $0.89 \pm 0.12^{\mathrm{A}}$ & $0.71 \pm 0.36$ & $0.64 \pm 0.03$ & $0.66 \pm 0.02$ & $1.0 \pm 0.06^{A}$ & $0.72 \pm 0.06$ \\
\hline LV FS & $46.5 \pm 8.0$ & $49.9 \pm 7.1$ & $39.8 \pm 10.5^{A}$ & $47.1 \pm 8.9$ & $45.4 \pm 3.4$ & $44.0 \pm 4.8$ & $26.3 \pm 5.1^{A}$ & $41.5 \pm 5.5$ \\
\hline TSPG, mmHg & NA & NA & $111.2 \pm 32.0$ & $95.2 \pm 34.5$ & NA & NA & $90.3 \pm 16.7$ & $82.0 \pm 14.5$ \\
\hline
\end{tabular}

HR, heart rate; LVIDd, LV internal dimension at end-diastole; LVIDs, LV internal dimension at end-systole; TSPG, trans-stenotic systolic pressure gradient as the difference between right carotid and left axillary artery systolic pressures. Results are expressed as mean \pm SEM. ${ }^{A P}<0.05$ between Fhl1 ${ }^{-1-}$ and $\mathrm{WT}^{-}$ at same point, and $P<0.05$ before and after TAC within the same group.

We were able to demonstrate that FHL1 interacts with this minimal $\mathrm{N} 2 \mathrm{~B}$ region of titin (Figure $3 \mathrm{C}$ ). Interaction between titin $\mathrm{N} 2 \mathrm{~B}$ and an HA control was not observed (Figure 3C). The colocalization of Raf1, MEK1/2, and ERK2 with titin N2B was also demonstrated by immunofluorescence staining of adult cardiomyocytes, which demonstrated their localization at the I band (Figure 3B).

FHL1 can modulate cardiac MAPK activation. The I band is a region long thought to be a "hotspot" for signaling (25); however, the consequences of these recently identified interactions on the signaling cascade remain unclear. Since ERK1/2 activation/phosphorylation is an endpoint of the Raf-mediated MAPK cascade, we sought to determine the consequences of loss of FHL1 on ERK1/2 activation. Fhl1/-- hearts showed a significant loss in ERK1/2 phosphorylation following TAC (Figure 3D). No significant differences in basal ERK1/2 phosphorylation were observed between WT and Fbl1 ${ }^{-/}$hearts (Figure 3D). Double KO/Gq hearts also demonstrated a significant reduction in ERK1/2 phosphorylation when compared with Gq hearts (Figure 3D). Again, no significant differences in basal cardiac ERK1/2 phosphorylation were observed between $\mathrm{Fbl1}^{-/}$, Gq transgenic, and double KO/Gq mice (Figure 3D). To determine whether FHL1 is sufficient to modulate ERK1/2 activation in cardiomyocytes, we assessed the effects of FHL1 overexpression on ERK1/2 activation in postnatal cardiomyocytes in vitro. Overexpression of FHL1 caused a significant increase in ERK1/2 phosphorylation compared with control infected cardiomyocytes (Figure 3E). No significant differences in Akt phosphorylation were observed between groups (Figure 3, D and E).

Based on the loss of ERK1/2 activation and hypertrophy (Figure $1 \mathrm{G}$ and Figure 3E) in Fhl1-/- hearts following TAC, we sought to determine the consequences of Fbl1 deficiency on markers of these parameters in isolated papillary cardiac muscles following acute stretch (Figure 4A), since it is not possible to directly assess ERK1/2 activation in these small muscles. ELK1, a transcriptional target of ERK1/2 activation (26), and ANF, a marker of stretch and hypertrophy (27), were assessed by quantitative PCR analysis in isolated papillary muscles stretched for 5 hours at $90 \%-95 \%$ $L_{\max }$ (muscle length at which the muscle develops the maximum force). ELK1 and ANF expression were significantly increased in stretched WT muscles, while no significant increases in ELK1 or ANF expression were observed in stretched Fbl1-deficient muscles (Figure 3F), demonstrating a loss of stretch-induced hypertrophic signaling responses.
Selective reduction of titin I band associated passive tension in isolated adult FHL1-deficient muscles after stretch. FHL1 interacts with the cardiac-specific N2B element of titin, which is part of the extensible I band region of titin that contributes to the myofibrillar passive/diastolic tension generated upon stretch (25). We assessed passive mechanical properties within isolated $\mathrm{Fbl1}^{-/-}$cardiac muscles by subjecting muscles to a maximum extension of $15 \%-20 \%$ stretch (Figure 4A). No significant differences in the cross-sectional area of muscle and sarcomere slack length were observed between Fbl1/-- and WT muscles (Figure 4B). However, $F_{b l 1^{-/}}$muscles displayed a significant reduction in diastolic stress (Figure 4C) and therefore increased compliance compared with controls. An increase in sarcomere length was also observed in $\mathrm{Fbl1}^{-/-}$ muscles when stretched to a diastolic stress of $20 \mathrm{kPa}$, compared with WT muscles (Figure 4B). Active mechanical properties, such as developed systolic stress, time to peak contraction, maximum rate of force production $(+\mathrm{df} / \mathrm{dt}$ maximum), $90 \%$ relaxation time, and maximum rate of force decline (-df/dt maximum) were not significantly different between $\mathrm{Fbl1}^{-/-}$and WT muscles at $L_{\max }$ (Figure 4C), suggesting a specific role for FHL1 in diastolic tension/function.

\section{Discussion}

Our studies have identified what we believe is a novel complex within the cardiomyocyte sarcomere that is involved in sensing biomechanical stress and plays a pivotal role in the early events leading to pathological cardiac hypertrophy. Fhl1 is a member of the newly identified FHL family, which has long been thought to play an important role in biomechanical stress responses involved in cardiac hypertrophy and disease in mice and humans (14-18). Our studies reveal that under conditions of in vivo pressure overload, Fhl1 deficiency in mice is sufficient to blunt hypertrophy and early progression to heart failure, while preserving cardiac function. Surprisingly, even though $\mathrm{Fbl1}^{-/-}$mouse hearts did exhibit some hypertrophy in the long-term TAC studies, this hypertrophy was not associated with a decrease in cardiac function and is suggestive of a beneficial form of hypertrophy reminiscent of physiological hypertrophy. In this regard, it is interesting to note that there are 2 other genetically altered mouse models that develop limited hypertrophy in response to chronic pressure overload ( 8 weeks of TAC), in which little or no deterioration in cardiac function is observed (28). The effects of Fbl1 deficiency on hypertrophy are not influenced by FHL2, since no differences in FHL2 protein expression were observed between WT and Fbl1 ${ }^{-/-}$hearts. Also, we previously demonstrated that Fbl2 ${ }^{-/-}$mice do 
A

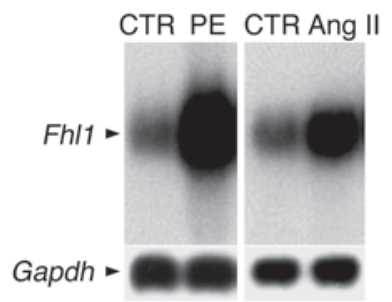

B

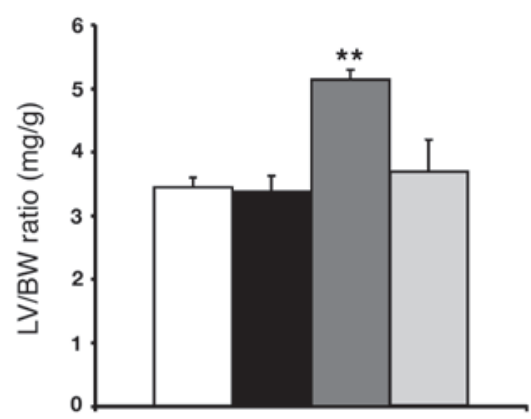

lacZ Gq

virus virus

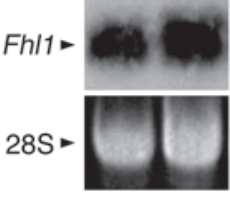

WT Gq+

FHL1 -

GAPDH -
C

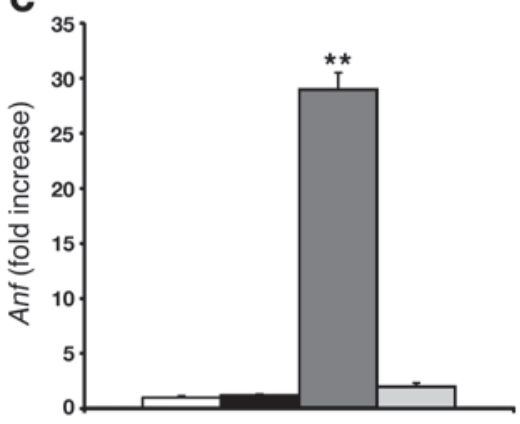

D
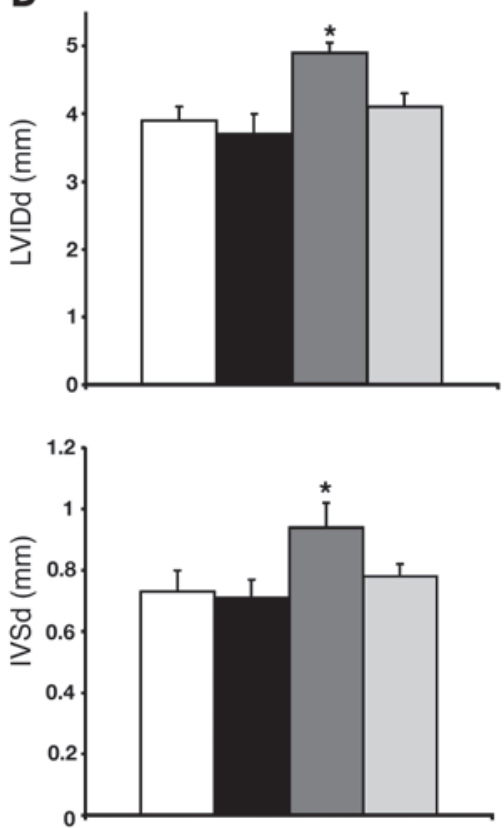
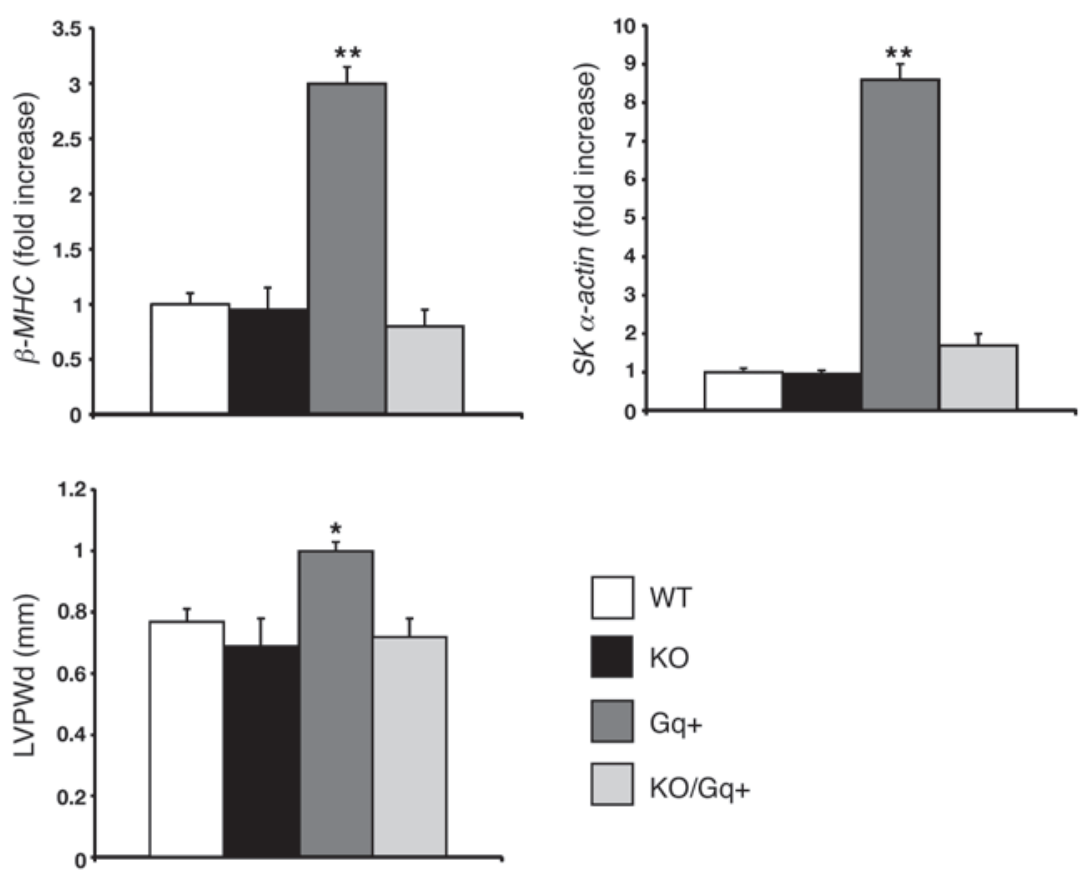
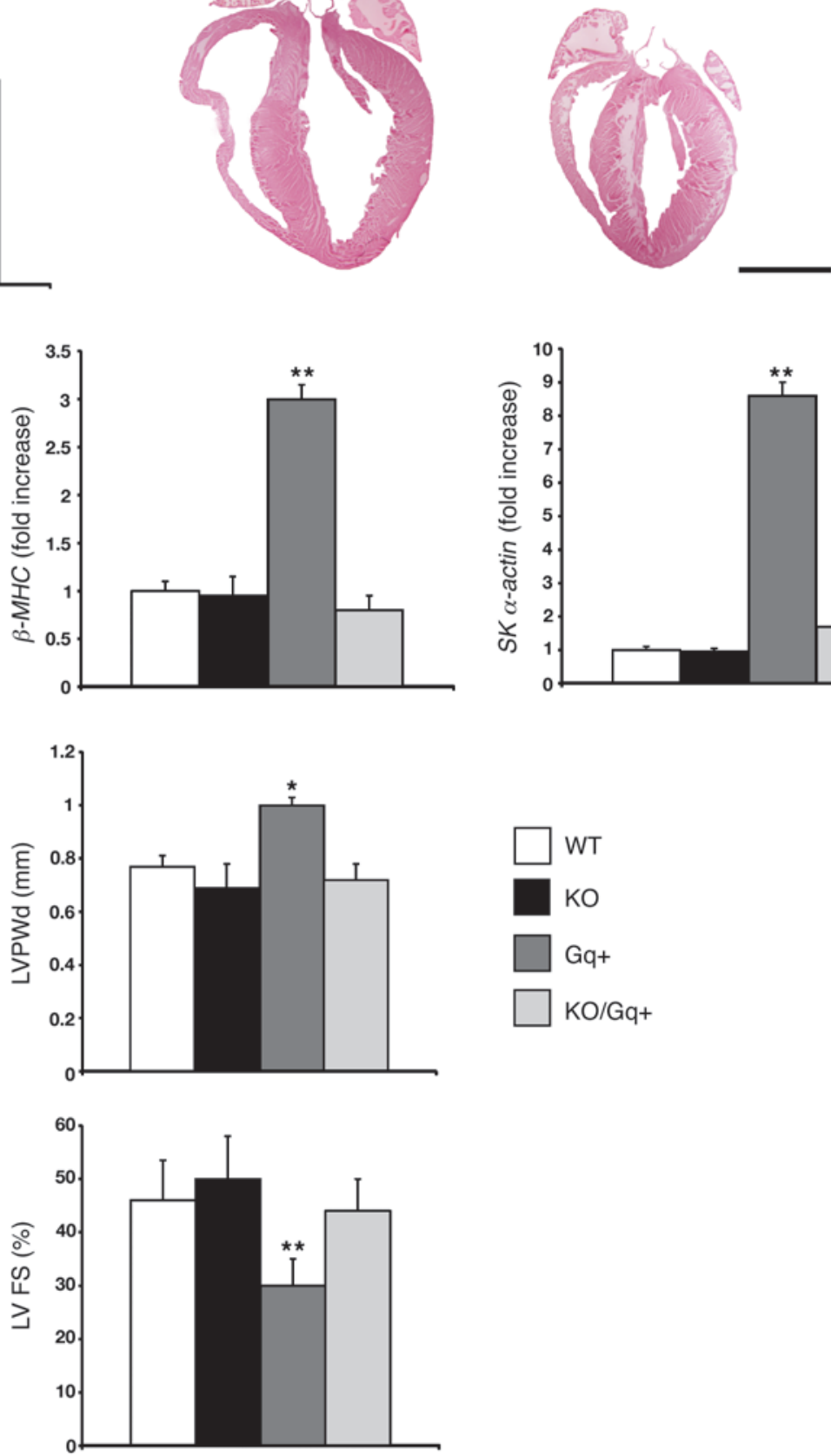

Figure 2

FHL1 is linked to the Gq signaling pathway in cardiomyocytes. (A) Fhl1 expression in cardiomyocytes following phenylephrine (PE) and angiotensin II treatment as well as viral Gq overexpression, using a constitutively active Gq virus and Gq transgenic mouse model. Left and middle: RNA $(10 \mu \mathrm{g})$ was electrophoresed from control and agonist-/viral-treated cardiomyocytes and analyzed using a cDNA probe spanning the Fh/1 coding region. GAPDH or $28 \mathrm{~S}$ RNA was used as a loading control. Right: Protein $(25 \mu \mathrm{g})$ from WT and Gq+ hearts at 12 weeks of age were subjected to 
SDS-PAGE and analyzed for FHL1 ( $32 \mathrm{kDa})$ protein using FHL1 antibodies. GAPDH ( $\sim 37 \mathrm{kDa})$ protein was used to normalize for loading. (B) LV weight/BW ratios in $\mathrm{WT}, \mathrm{KO}, \mathrm{Gq}+$, and double $\mathrm{KO} / \mathrm{Gq}+$ mice at 12 weeks of age $(n=6)$. Paraffin sections from $\mathrm{Gq}+$ and $\mathrm{KO} / \mathrm{Gq}+$ hearts were stained for nuclei and cytoplasm, respectively, with hematoxylin and eosin. Scale bar: $2.5 \mathrm{~mm}$. (C) Anf, $\beta-M H C$, and skeletal $\alpha$-actin RNA expression in WT, KO, Gq+, and KO/Gq+ LVs $(n=4)$ were normalized for Gapdh expression and presented as fold induction relative to WT. (D) Echocardiographic measurements obtained from WT, KO, Gq+, and KO/Gq+ LVs at 12 weeks of age. LVIDd, LV internal dimension at end-diastole. ${ }^{*} P<0.05 ;{ }^{* \star} P<0.01$.

not exhibit any significant differences in the hypertrophic response following TAC when compared with WT controls (29). Our studies further demonstrate that FHL1 plays a pivotal role in active relaxation, since $\mathrm{Fbl1} \mathrm{1}^{-/-}$hearts exhibited increased $-\mathrm{dP} / \mathrm{dt}$ maximum and relaxation time constant as well as preserved LV end-diastolic pressure after TAC when compared with WT hearts. Interestingly, loss of FHL1 also results in beneficial effects on the systolic parameters within the heart after pressure overload.

FHL1 is a part of what is, to our knowledge, a novel complex within the sarcomeric central I band of titin formed by the direct interaction between FHL1 and the N2B region of titin. Titin is the largest known cytoskeletal protein and primarily functions as a molecular spring to provide myofibrils with elasticity (25). Cardiac titin contains a unique cardiac-specific N2B element within the sarcomeric I band that is thought to act as a biomechanical sensor and contributes to myofibrillar passive tension (diastolic function) generated upon stretch $(25,30)$. This is consistent with studies in titin $\mathrm{N} 2 \mathrm{~B}$-deficient mice, which demonstrate that the $\mathrm{N} 2 \mathrm{~B}$ region of titin affects diastolic but not systolic function of the heart (31). Multiple mechanisms of titin-based stretch sensing and signaling pathways have been proposed within the central I band $(5,32)$. However, our studies demonstrate an in vivo role for the titin N2B binding partner, FHL1, in diastolic function and biomechanical stress responses involved in pressure overload-induced hypertrophy.

Our data strongly suggest that FHL1-mediated pathways lie downstream of Gq signaling, since Fbl1 deficiency in mice completely prevents Gq-mediated cardiomyopathy. In addition, GPCR agonists or overexpression of constitutively active Gq upregulates FHL1 expression in postnatal cardiomyocytes in vitro and mouse hearts in vivo, respectively. FHL1 also intersects with the Gq signaling pathway by directly interacting with downstream signaling components Raf1, MEK2, and ERK2. Our studies further demonstrate a role for FHL1 as an endogenous positive regulator of Raf-1/MEK/ERK-mediated signaling. This was most clearly evidenced when FHL1 overexpression was sufficient to increase cardiomyocyte ERK1/2 phosphorylation and by the blunted response to ERK1/2 phosphorylation in $\mathrm{Fbl1}^{-/-}$cardiac muscles following TAC and in the background of $\mathrm{Gq}$ overexpression in vivo. The $\mathrm{Gq}$ signaling pathway has been shown to target ERK activation under conditions of in vivo pressure overload (33).

FHL1 could also fulfill a scaffolding function, since direct interactions between FHL1 and Raf-mediated MAPK cascade converge at the N2B region of titin. Scaffolding proteins are dynamic entities that can regulate signal transduction pathways by tethering molecules together in a subcellular location and stimulus- specific manner, serving as the molecular backbones for signaling complex assembly $(34,35)$. Studies on proteins such as kinase suppressor of Ras (KSR) have identified an important role for scaffold proteins in regulating MAPK signaling $(36,37)$. There are several proposed functions for MAPK scaffold proteins, which include activation of kinases, regulation of the intensity of signaling responses, recruitment or movement of a complex to a location, consolidation or sequestration of proteins to regulate protein interactions, or control of protein subcellular localization (38). KSR functions by regulating the intensity and duration of Raf/MEK/ERK signaling, which could alter cell fate (38). Our results suggest that FHL1 may not only be important in subcellular localization of the MAPK complex at the I band but also may enhance intensity and duration of MAPK signaling to facilitate hypertrophic signaling, since increased endogenous interactions were observed between FHL1 and the MAPK cascade components following TAC (a stimulus known to increase ERK signaling). Although several studies support the role of Raf1 and MEK1/2 in cardiac hypertrophy (39-42), a recent study that employed gene $\mathrm{KO}$ and transgenic approaches suggested that ERK-mediated phosphorylation is not essential for cardiac hypertrophy (42). Results of the latter study, performed in gene KO models (conventional ERK2 heterozygous mice and conventional ERK1 homozygous mice), as the authors point out, leave open the possibility that the level of residual ERK1/2 activity in these models could be sufficient to mount a hypertrophic response and insufficient to accurately determine the effects of a complete loss of ERK1/2 signaling in hypertrophy. This study, which involved transgenic overexpression of the MAPK phosphatase DUSP6 also suggest that ERK1/2 phosphorylation is not involved in hypertrophy (42). However, it is also not clear whether in vivo overexpression of DUSP6 could influence other pathways, which could compensate for the loss of ERK1/2 activity. Our studies demonstrate that changes in sarcomeric extensibility and intracellular signaling responses related to ERK1/2 signaling may both contribute to the abrogration of hypertrophy in $\mathrm{Fbl1}^{-/-}$mice.

$\mathrm{Fhl1}^{-/-}$muscles display increased compliance. Although no significant differences in slack sarcomere length were observed between WT and $\mathrm{Fbl1}^{-/-}$muscles, an increase in sarcomere length/extensibility was observed in Fbl1/-- muscles when compared with WT muscles at a similar stress. Since the I band region is the extensible region of the sarcomere and FHL1 interacts with titin N2B, these studies further point to a direct role for FHL1 in I band-associated sarcomere extensibility. These findings are different from the

\section{Table 2}

Quantitative $\beta$-galactosidase assays for domain interactions between FHL1 and Raf1, MEK1/2, and ERK2

\begin{tabular}{lcclcc}
\hline & & & & & \\
& Raf1 & MEK1 & MEK2 & ERK2 & ERK2 (TYDD) \\
FHL1 (4.5 LIM) & $49 \pm 2.6^{\mathrm{A}}$ & $1 \pm 0.3$ & $33 \pm 3.1^{\mathrm{A}}$ & $39 \pm 3.8^{\mathrm{A}}$ & $58 \pm 2.6^{\mathrm{A}, \mathrm{B}}$ \\
FHL1 (3.5 LIM) & $25 \pm 1.2$ & $1 \pm 0.2$ & $18 \pm 2.4$ & $26 \pm 1.5$ & $28 \pm 1.3$ \\
FHL1 (2 LIM) & $20 \pm 2.6$ & $1 \pm 0.4$ & $19 \pm 2.4$ & $19 \pm 2.7$ & $26 \pm 1.2$ \\
FHL1 (1.5 LIM) & $1 \pm 0.2$ & $2 \pm 0.6$ & $1 \pm 0.2$ & $1 \pm 0.3$ & $1 \pm 0.3$ \\
FHL1 (Kyot2) & $1 \pm 0.3$ & $1 \pm 0.3$ & $1 \pm 0.2$ & $1 \pm 0.2$ & $1 \pm 0.3$ \\
\hline
\end{tabular}

One unit of $\beta$-galactosidase was defined as the amount of enzyme that hydrolyzes 1 $\mu \mathrm{mol}$ of ortho-nitrophenyl- $\beta$-D-galactopyranoside (ONPG) to O-nitrophenol and D-galactose per minute per cell. The results from 4 separate measurements are expressed as mean \pm SEM. ${ }^{A} P<0.01$, full-length FHL1 (4.5 LIM) versus truncated FHL1 forms. ${ }^{B} P<0.01$, interaction of full-length FHL1 (4.5 LIM) and ERK2 versus full-length FHL1 (4.5 LIM) and ERK2 (TYDD). 
A

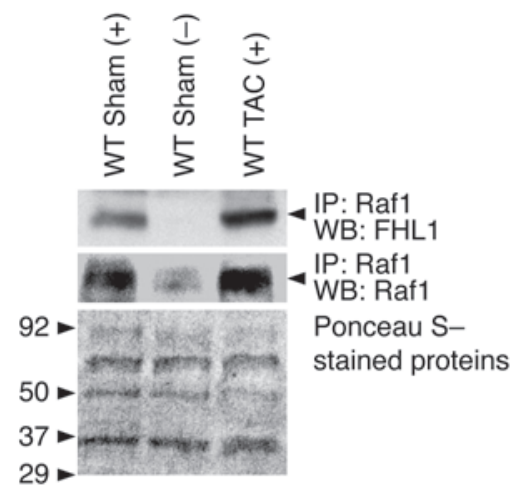

B
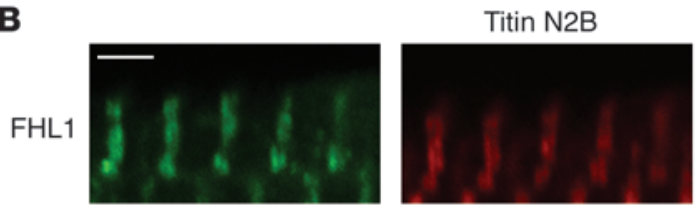

Raf1
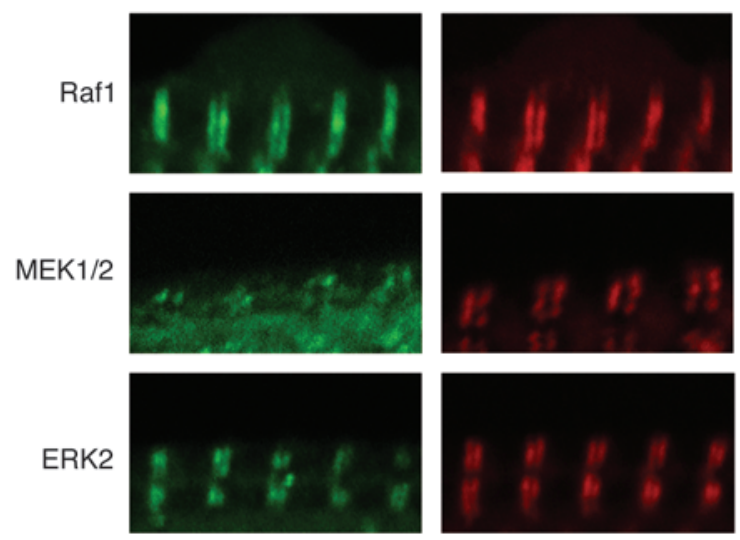
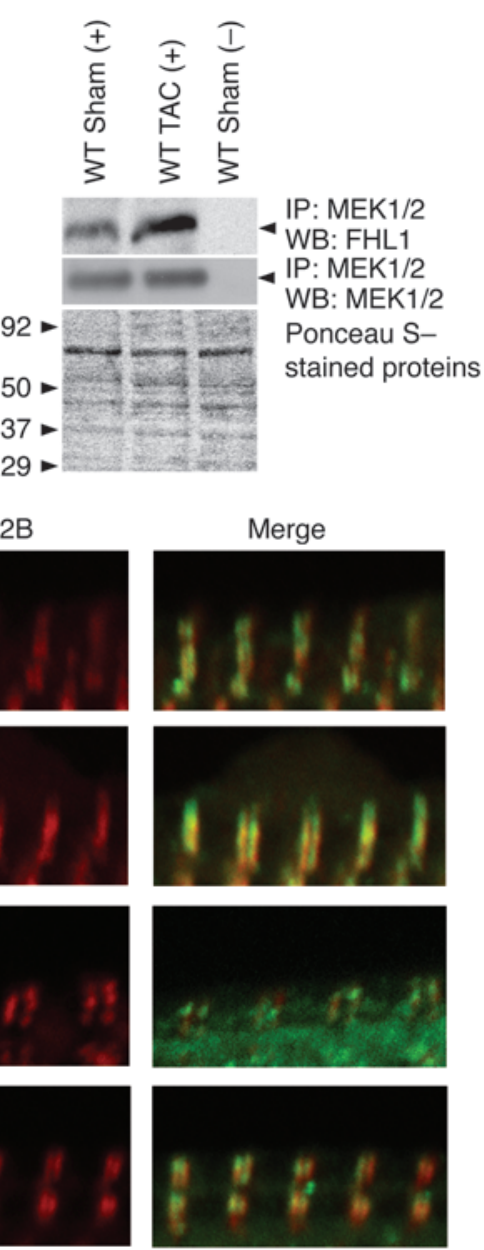
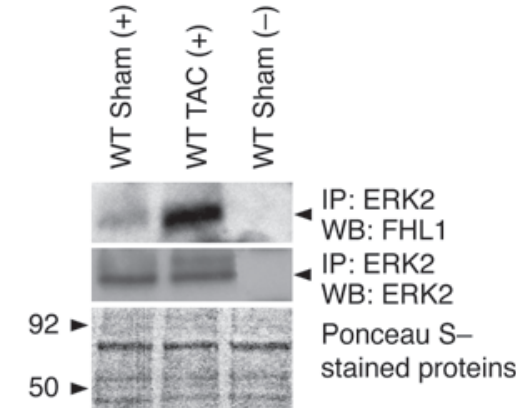

$37-$

29

C

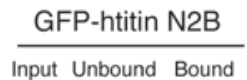

HA-FHL2

HA-FHL1

HA-ctrl

IP: $\alpha$ HA WB: $\alpha$ GFP

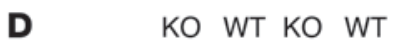

phospho-

ERK $1 / 2$

Total ERK $1 / 2$

phospho-

Akt

Total Akt

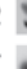

KO WT KO WT
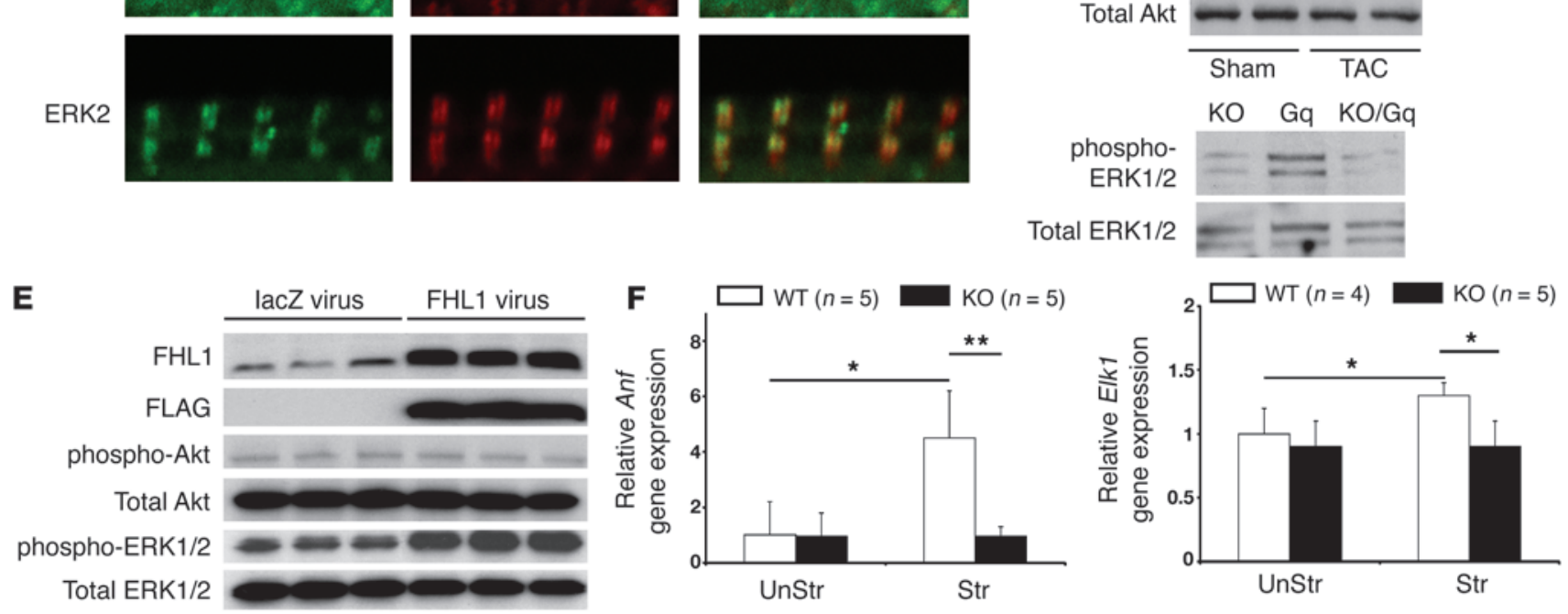

\section{Figure 3}

FHL1 associates with and affects the MAPK pathway. (A) Raf1 (left), MEK1/2 (middle), and ERK2 (right) were immunoprecipitated from 8-weekold adult mouse heart extracts $(500 \mu \mathrm{g})$ following sham and TAC (1 week) operation and western blot analysis for FHL1 ( $\sim 32 \mathrm{kDa})$, Raf1 ( $80 \mathrm{kDa})$, MEK1/2 ( $47 \mathrm{kDa})$, and ERK2 (44 kDa) proteins as indicated. Plus and minus symbols indicate the presence and absence of antibody. Molecular weight standards are indicated on Ponceau S-stained membranes. (B) Adult cardiomyocytes were stained with antibodies against FHL1, Raf1, MEK1/2, ERK2, and titin N2B. Merged images of doubly stained cardiomyocytes are shown. Scale bar: $2 \mu \mathrm{m}$. Double arrows indicate colocalization. (C) COS cells were cotransfected with GFP-htitinN2B and HA-tagged FHL1 or FHL2 or control. Coimmunoprecipitation was carried out with an anti-HA antibody, followed by detection of the GFP fusion protein ( $~ 80 \mathrm{kDa})$ with GFP antibodies. "Input" indicates non-fractionated protein prior to coimmunoprecipitation assay. (D) Western blot analysis of phosphorylated and total ERK1/2 (42 and $44 \mathrm{kDa})$ and Akt (60 kDa) in WT and $\mathrm{KO}$ adult mouse hearts after sham or TAC operation for 7 days (top); $\mathrm{KO}, \mathrm{Gq}+$, and $\mathrm{KO} / \mathrm{Gq}+$ mouse hearts (12 weeks old; bottom); and (E) lacZ and FLAG-FHL1 viral treatment of neonatal cardiomyocytes for 24 hours. Expression of the FLAG-FHL1 virus was confirmed with FLAGand FHL1-specific antibodies. (F) RT-PCR analysis of Anf and Elk1 expression in isolated WT and KO muscles $(n=5)$ before stretch (UnStr) and after stretch (Str). ${ }^{*} P<0.05 ;{ }^{* *} P<0.01$. 
A

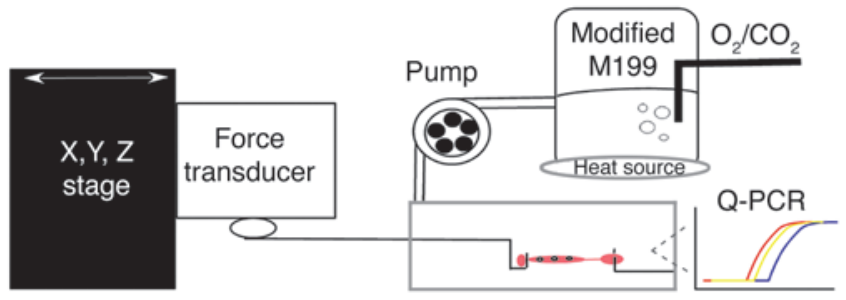

Culture chamber
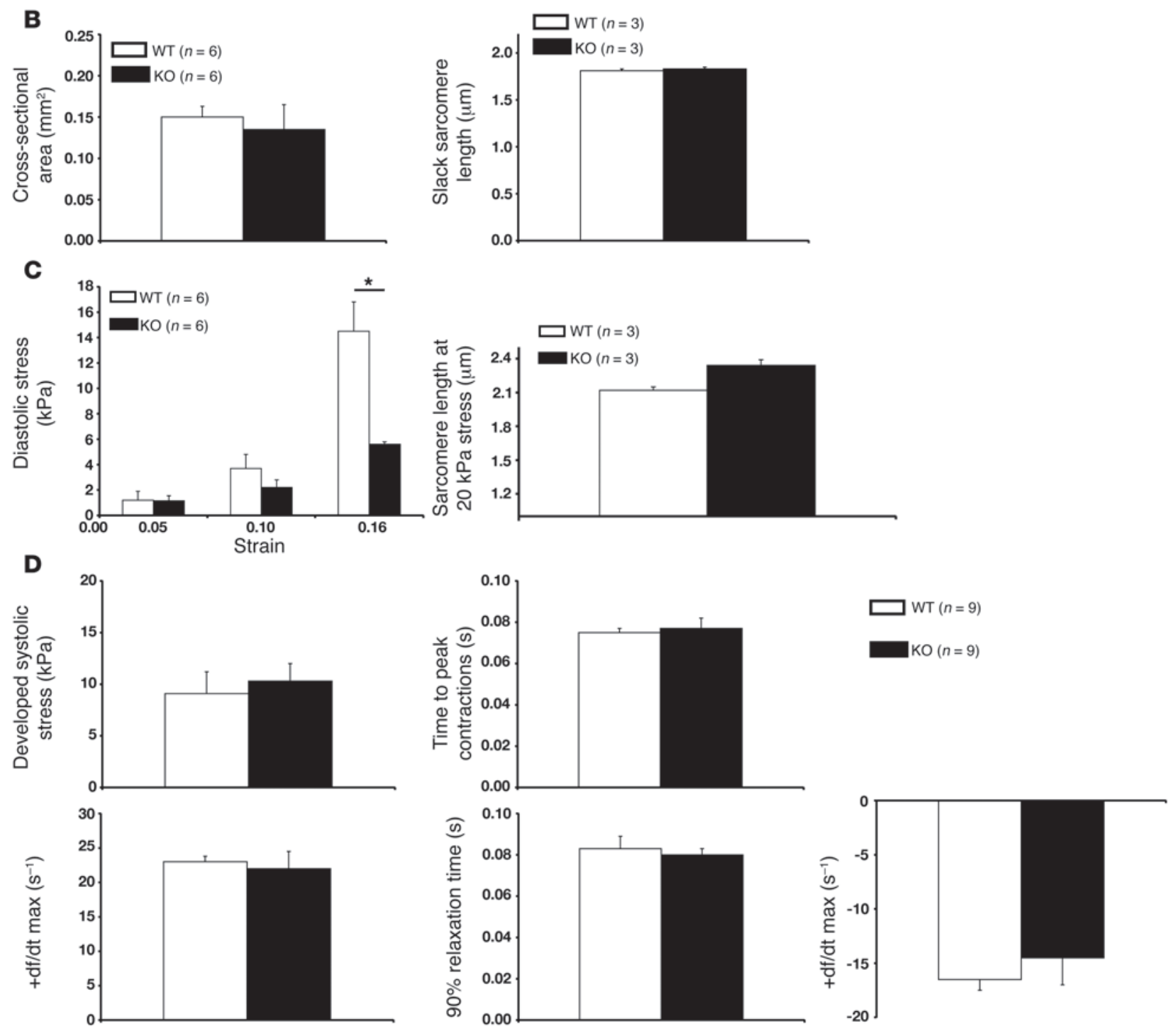

Figure 4

Muscle mechanics within isolated adult WT and Fh/1-deficient cardiac muscles following stretch. (A) Schematic representation of isolated adult RV papillary mouse muscle from 8- to 12-week-old mice in a tissue chamber. Specimens were stretched for 5 hours to a maximum extension of $15 \%-20 \%\left(90 \%-95 \% L_{\max }\right)$. Control muscles were left in the system for the same period of time at slack length. The system was used to analyze load-inducible hypertrophic markers as well as passive and active stresses as a function of passive stretch. (B) Quantitative assessment of cross-sectional area and slack sarcomere lengths in WT and Fhl1-deficient muscles. (C) Passive tensile stress (diastolic stress) of RV papillary muscles in WT and KO muscles before and after stretch. Sarcomere lengths in WT and KO muscles at $20 \mathrm{kPa}$ stress. ${ }^{*} P<0.05$. (D) Active mechanical properties were assessed in isolated WT and KO muscles as a function of stretch. 


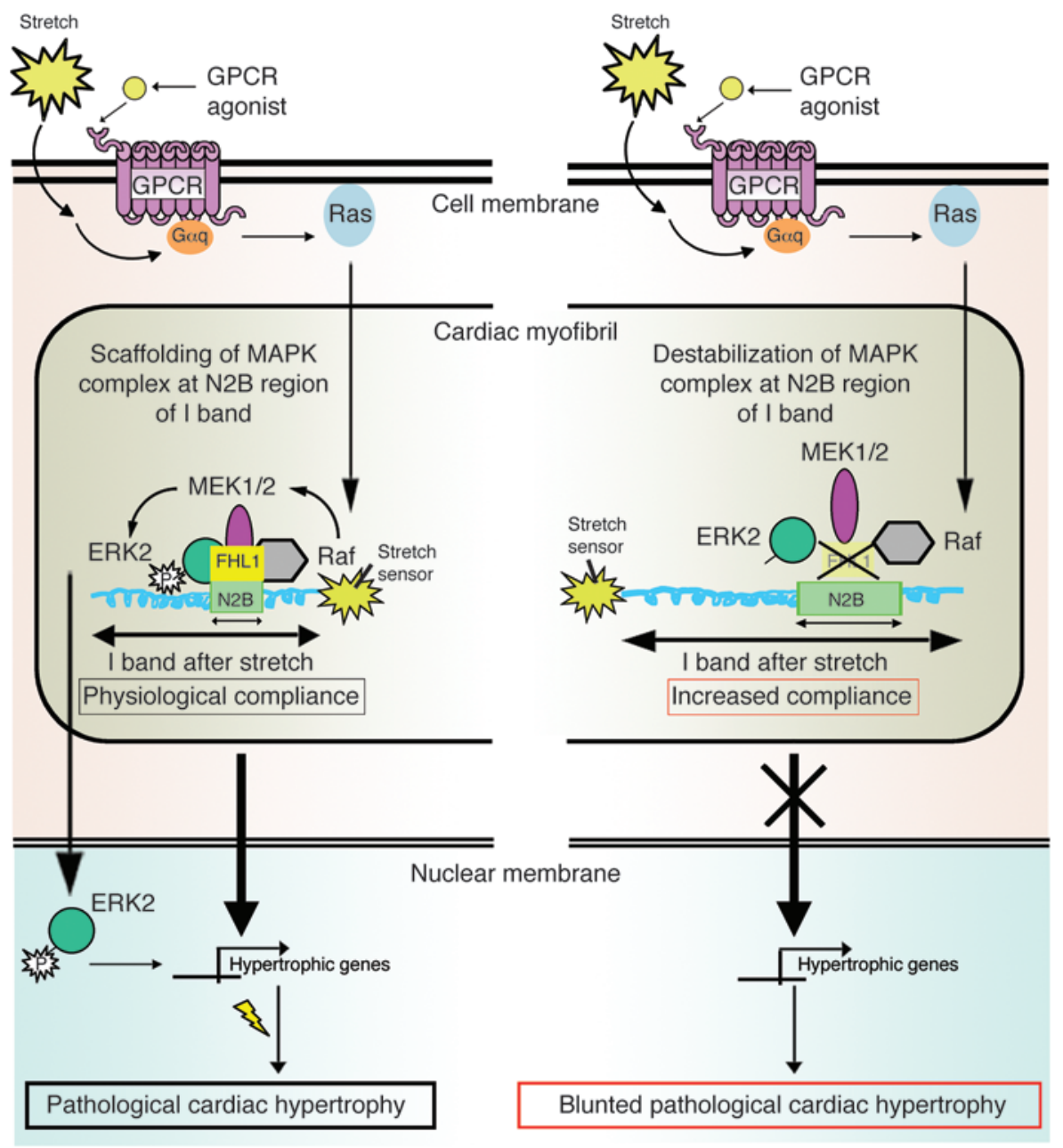

\section{Figure 5}

Working model for the pathways that link defects in the MAPK/FHL1/titin complex to cardiac sarcomere extensibility and hypertrophic signaling. Left: FHL1 is a component of the stretch sensor at the I band that acts to sense stretch or agonists induced by GPCR $(\mathrm{Gq})$ signaling to restrict or lock the range at which physiological sarcomere length can extend following stretch and/or to scaffold Gq/stress-induced interactions of MAPK components at titin N2B in order to mediate ensuing hypertrophic signaling, which can lead to pathological cardiac hypertrophy. Right: Loss of FHL1 leads to unlocking and/or destabilization of the macromolecular MAPK/FHL1/titin complex in cardiomyocytes, thereby resulting in increased passive elasticity and sarcomere extensibility and/or loss of the ability to scaffold $\mathrm{Gq} /$ stress-induced interactions of MAPK at the I band, resulting in reduced hypertrophic signaling and a blunted response to cardiac hypertrophy. results of studies in MLP- and titin N2B-deficient mice, in which the reduced passive tension and basal cardiac muscle defects were likely due to shortened sarcomere slack lengths $(4,31,32)$. Extracellular collagen is one of the major contributors to myocardial diastolic tension at longer sarcomere lengths (43). However, no obvious changes in collagen content, as assessed by Masson's trichrome stain, were detected in $\mathrm{Fbl1}^{-/-}$hearts compared with those of controls (our unpublished observations), further pointing to an influence of titin on passive mechanics in $\mathrm{Fbl1}^{-/-}$mice.

In conclusion, our studies propose a working hypothesis whereby FHL1 plays an important role in the general mechanism of pathological hypertrophy by sensing biomechanical stress responses in the sarcomere via the titin $\mathrm{N} 2 \mathrm{~B}$ region, thus leading to changes in MAPK- and titin-mediated responses, which are important for the transition to pathological hypertrophy (Figure 5). The N2B region of titin extends mostly toward the upper limit of the physiological sarcomere length of cardiac muscle $(25,44)$. Our findings highlight what we believe is a novel role for FHL1 in modulating titin N2B function and sarcomere extensibility at the upper limits of the physiological sarcomere length, a state that could be physiologically reached in vitro through acute stretch and in vivo by pressure overload. Since Fbl1 deficiency is sufficient to prevent the deleterious effects following $\mathrm{Gq}$ overexpression in vivo, these studies further suggest that the MAPK/FHL1/titin complex is a key target of Gq signaling. A recent study has identified a titin N2B mutation associated with hypertrophic cardiomyopathy in a region that specifically binds and modulates FHL affinity (45), suggesting the possibility that destabilization of the macromolecular titin/I band complex including FHL1 could also potentially underlie genetic forms of cardiomyopathy. A better understanding of the role of FHL1 in titin and Gq/MAPK signaling in striated muscle in vivo could be of critical importance in developing strategies (i.e., inhibitors of FHL1) to abolish the deleterious effects of pathological hypertrophy in human cardiac disease.

\section{Methods}

Generation of gene-targeted and transgenic mice. Fhl1 genomic DNA was isolated from a 129-SV/J mouse genomic DNA library (Stratagene). PCRbased mutagenesis was used to convert the approximately 350-bp sequence between 2 bp $5^{\prime}$ of the translation start codon ATG in Fbl1 exon 1 (the first coding exon) and $2 \mathrm{bp} 5^{\prime}$ of the EcoRI site in intron 1 to a SalI site. The deleted 350-bp fragment would contain part of Fbl1 exon 1 and part of intron 1. A LacZ/pGKneo cassette was inserted into the Sall site using Xhol, thereby destroying the Sall site. The targeting construct was linearized with SstII before electroporation into RI ES cells. G418-resistant ES clones were screened for homologous recombination by BamHI digestion, followed by Southern blot analysis, as previously described (8). Two independent homologous recombinant ES clones were microinjected into C57BL/6J blastocysts and transferred into pseudopregnant recipients. Chimeric ani- 
mals resulting from the microinjection were bred with Black Swiss mice to generate germ line-transmitted agouti heterozygous female mice $\left(\mathrm{Fbll}^{+/-}\right)$ or hemizygous male (Fbl1-/y) mice. Fbl1 localizes at the X chromosome, and because large numbers of WT littermate and $\mathrm{Fbl1}^{-/-}$mice could be attained per litter, all of our experiments were performed on male mice. Offspring from Fbl1 intercrosses were genotyped by PCR analysis. PCR analysis was performed on tail DNA from mouse offspring from Fbl1 intercrosses by using Fbl1 WT allele- (forward, ACCATGTCGG AGAAGTTCGACT; reverse, AGGCTGCAGGTATACACCTGAA) as well as Fhl1 KO allele-specific primers (forward, CCTCTGAGTCAGCAGCGTGATT; reverse, AGATGAAACGCCGAGTTAACGC) using standard procedures. Genotypes for Gq mice were determined using tail DNA and Gq transgene-specific primers (forward, CAGGACTTCACATAGAAGCC; reverse, CGTGAAGATGTTCTGATACACC) using standard procedures. Fhl1 hemizygous KO mice (Fbl1-/y) were also mated with Gq-40-overexpressing mice (22) to generate hemizygous FHL1 offspring carrying the Gq transgene (KO/Gq). F1 KO/Gq mice were bred to heterozygous Fbl1 littermates lacking the transgene in order to obtain F2 mice with 5 different genotypes - WT, Gq gain-of-function transgenic $\left(\mathrm{Gq}^{+}\right)$, Fbl1 hemizygous $\mathrm{KO}$, and the combinatorial genotypes (Fbl1 hemizygous $\mathrm{KO} / \mathrm{Gq}^{+}$and Fbl1 heterozygous $\mathrm{KO} / \mathrm{Gq}^{+}$) - within the same litter. Comparisons were only made within litters, which contained $\mathrm{KO}, \mathrm{Gq}^{+}, \mathrm{KO} / \mathrm{Gq}^{+}$, and age-matched WT littermate controls, and thus utilizing only male animals made the breeding process much easier. All animal procedures were approved by the UCSD Animal Care and Use Committee.

Protein blot analysis. Total protein extracts were prepared as previously described (8). Polyclonal antibodies to FHL1 were produced in rabbits immunized with a synthetic KLH-conjugated peptide corresponding to mouse FHL1 protein (DPLQGKKYVQKDGR) and were used for immunodetection of FHL1 (1 $\mu \mathrm{g} / \mathrm{ml}$; Chemicon International). Immunodetection of FHL2 (1:1000; a kind gift from Beat Schäfer, Institute of Cell Biology, ETH Hönggerberg, Zurich, Switzerland), glyceraldehyde-3-phosphate dehydrogenase (1:1000; Cell Signaling Technology), elongation factor- $1 \alpha(1 \mu \mathrm{g} / \mathrm{ml}$; Chemicon International), phospho-ERK1/2 (1:1000; Cell Signaling Technology), total ERK1/2 $(0.5 \mu \mathrm{g} / \mathrm{ml}$; Upstate USA Inc.), phospho-Akt (Ser473) (1:1000; Cell Signaling

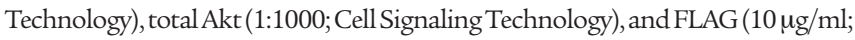
Sigma-Aldrich) was performed as previously described (27).

Histological and morphometric analyses. Cardiac sections (8 $\mu \mathrm{m}$ thick) were stained with hematoxylin and eosin as previously described (8). Cardiac sections were immunostained with fluorescein-conjugated wheat germ agglutinin (Invitrogen) and stained for DNA with Hoechst 33342 to measure myocyte area at $\times 400$ magnification (Olympus).

In vivo pressure overload model. Adult mice (8-10 weeks old) were anesthetized with ketamine/xylazine, and TAC was performed as previously described (46).

Hemodynamic and blood pressure measurements. Please see Supplemental Methods for details.

RNA analysis. Total RNA was extracted from LVs, cardiomyocytes, and papillary muscles using TRIzol (Invitrogen) or RNeasy (Qiagen). Dot blot and northern blot analyses were performed as previously described $(14,47)$. Quantitative PCR was performed using the ABI 7700 real-time thermal cycler using standard procedures.

Echocardiography. Adult mice were anesthetized with $1 \%$ isoflurane and subjected to echocardiography as previously described (48).

Cardiomyocyte cultures and treatments/infections. Cardiomyocytes were prepared from 1- to 2-day-old neonatal rats as previously described (27) and treated with phenylephrine $(0.1 \mathrm{mM})$ or angiotensin II $(100 \mathrm{nM})$ for $24 \mathrm{~h}$. Cardiomyocytes were treated with PD98059 $(50 \mu \mathrm{M} / \mathrm{l}), \mathrm{SB} 203580(10 \mu \mathrm{M})$, or wortmannin $(10 \mu \mathrm{M} / \mathrm{l})$ in the presence of phenylephrine $(0.1 \mathrm{mM})$ or angiotensin II (100 nM). Adenovirus vectors containing the lac $Z$ and mouse Fbl1 cDNAs were prepared and used at multiplicity of infection $25-50 \mathrm{pfu} / \mathrm{cell}$ as previously described (27). The constitutively active Gq adenovirus (Q209L) was previously generated and was used in cardiomyocytes as previously described (49).

Yeast 2-hybrid assays. Yeast 2-hybrid and $\beta$-galactosidase assays were carried out according to manufacturer's instructions (Clontech). To assay the interaction between FHL1 with Raf-1, MEK1, MEK2, ERK2, and ERK2 (TYDD), the coding sequences for full-length FHL1 (4.5 LIM), the small isoform of FHL1 (KyoT2), and the deletion mutants FHL1 (3.5 LIM), FHL1 (2 LIM), and FHL1 (1.5 LIM) were generated by PCR and were subcloned into the pLexA bait vector (50). The coding sequences for Raf1, MEK1, MEK2, ERK2, and ERK2 (TYDD) were generated by PCR and subcloned into the vector PACT2 (Clontech). ERK2 (TYDD) was generated by PCR-based mutagenesis (Quikchange; Stratagene). Two plasmids were cotransformed into the yeast strain L40 and assayed for $\beta$-galactosidase activity and for growth on selection plates lacking the amino acids tryptophan, leucine, and histidine (51). Four independent positive L40 yeast colonies per protein-protein interaction were assessed for $\beta$-galactosidase activity using the ortho-nitrophenyl- $\beta$-D-galactopyranoside-driven (ONPGdriven) liquid culture method as described by the manufacturer (Clontech). Primers and templates for PCR are described in Supplemental Table 3.

Immunoprecipitation assays. Protein extracts were isolated and prepared as previously described (24). Raf1 (Santa Cruz Biotechnology Inc.), MEK1/2 (Cell Signaling Technology), and ERK2 (BD) antibodies were used for immunoprecipitation according to the manufacturers' instructions, followed by SDS-PAGE and immunodetection of FHL1 $(1 \mu \mathrm{g} / \mathrm{ml})$. For immunoprecipitation assays in COS-1 cells, plasmids encoding HA-tagged DRAL/FHL2 (HA-DRAL), HA-tagged FHL1 (HA-FHL1), and HA-tagged p62 control were cotransfected with GFP-tagged human cardiac titin N2B us3 (residues 3,794-4,064 of the cardiac N2B isoform; NCBI accession no. X90568; ref. 52) into COS-1 cells. The HA-tagged DRAL/FHL2 and FHL1 constructs have been previously described (24). Cells were harvested 24-48 hours later and were immunoprecipitated with monoclonal HA antibodies (1:100; Roche) and processed for SDS-PAGE and GFP (1:1000; Roche) immunodetection as previously described (24).

Immunofluorescence microscopy. Adult cardiomyocytes were isolated (53) and incubated in a relaxation buffer (24), fixed with 4\% paraformaldehyde, and stained with primary antibodies against FHL1 (1:50), Raf1 (1:200; Santa Cruz Biotechnology Inc.), ERK2 (1:100; BD), MEK1/2 (1:25; Cell Signaling Technology), and titin N2B (1:2; a kind gift from Mathias Gautel, King's College London, London, United Kingdom). Cells were then stained with secondary antibodies (1:250; Invitrogen), followed by visualization of signals by confocal imaging (BioRad Radiance 2000).

In vitro cardiac muscle model of load-induced hypertrophy. Please see Supplemental Methods for details.

Sarcomere length analysis. Mechanically unloaded RV papillary muscles were fixed with $4 \%$ paraformaldehyde in relaxing solution for 1 hour at $4{ }^{\circ} \mathrm{C}$. Muscle cryosections ( $5 \mu \mathrm{m}$ thick) were stained with primary antibodies against sarcomeric $\alpha$-actinin (1:200; Sigma-Aldrich), followed by secondary antibody staining (1:400; Invitrogen). Sarcomere lengths were visualized by confocal microscopy (BioRad Radiance 2000) and measured by FFT analysis (MATLAB) for sarcomere periodicity.

Statistics. Data presented in the text and figures are expressed as mean values \pm standard error of the mean. Significance was evaluated by the 2-tailed Student's $t$ test or repeated-measures ANOVA. Statistical analysis for muscle mechanics data is described in detail in the Supplemental Methods. $P<0.05$ was considered statistically significant.

\section{Acknowledgments}

We thank Mathias Gautel and Beat Schäfer for providing us with titin N2B and FHL2 antibodies, respectively. We also thank Katherine Banares for technical assistance. This work was supported by 
grants from the NIH (to J. Chen) and the National Health Research Institutes (to P.-H. Chu). F. Sheikh was the recipient of an American Heart Association (AHA) postdoctoral fellowship and is currently the recipient of an AHA National Scientist Development Grant. S. Lange is the recipient of a Muscular Dystrophy Association Development Grant. A.A. Domenighetti is the recipient of a Swiss National Science Foundation postdoctoral fellowship (PA00A3-115378).
Received for publication November 9, 2007, and accepted in revised form September 24, 2008.

Address correspondence to: Ju Chen, Department of Medicine, University of California, San Diego, 9500 Gilman Drive, La Jolla, California 92093, USA. Phone: (858) 822-4276; Fax: (858) 822-1355; E-mail: juchen@ucsd.edu.
1. Arber, S., et al. 1997. MLP-deficient mice exhibit a disruption of cardiac cytoarchitectural organization, dilated cardiomyopathy, and heart failure. Cell. 88:393-403.

2. Arimura, T., et al. 2004. A Cypher/ZASP mutation associated with dilated cardiomyopathy alters the binding affinity to protein kinase C. J. Biol. Chem. 279:6746-6752.

3. Geier, C., et al. 2003. Mutations in the human muscle LIM protein gene in families with hypertrophic cardiomyopathy. Circulation. 107:1390-1395.

4. Knoll, R., et al. 2002. The cardiac mechanical stretch sensor machinery involves a $\mathrm{Z}$ disc complex that is defective in a subset of human dilated cardiomyopathy. Cell. 111:943-955.

5. Linke, W.A. 2008. Sense and stretchability: The role of titin and titin-associated proteins in myocardial stress-sensing and mechanical dysfunction. Cardiovasc. Res. 77:637-648.

6. Pashmforoush, M., et al. 2001. Adult mice deficient in actinin-associated LIM-domain protein reveal a developmental pathway for right ventricular cardiomyopathy. Nat. Med. 7:591-597.

7. Vatta, M., et al. 2003. Mutations in Cypher /ZASP in patients with dilated cardiomyopathy and left ventricular non-compaction. J. Am. Coll. Cardiol. 42:2014-2027.

8. Zhou, Q., et al. 2001. Ablation of Cypher, a PDZLIM domain Z-line protein, causes a severe form of congenital myopathy. J. Cell Biol. 155:605-612.

9. Brancaccio, M., et al. 2002. Melusin, a muscle-specific integrin $\beta 1$-interacting protein, is required to prevent cardiac failure in response to chronic pressure overload. Nat. Med. 9:68-75.

10. Frey, N., et al. 2004. Mice lacking calsarcin-1 are sensitized to calcineurin signaling and show accelerated cardiomyopathy in response to pathological biomechanical stress. Nat. Med. 10:1336-1343.

11. Lee, S.M., et al. 1998. Chromosomal mapping, tissue distribution and cDNA sequence of fourand-a-half LIM domain protein 1 (FHL1). Gene. 216:163-170.

12. Cottle, D.L., et al. 2007. FHL3 binds MyoD and negatively regulates myotube formation. J. Cell Sci. 120:1423-1435.

13. Johannessen, M., Moller, S., Hansen, T., Moens, U., and Van Ghelue, M. 2006. The multifunctional roles of the four-and-a-half-LIM only protein FHL2. Cell. Mol. Life Sci. 63:268-284.

14. Chu, P.H., Ruiz-Lozano, P., Zhou, Q., Cai, C., and Chen, J. 2000. Expression patterns of FHL/SLIM family members suggest important functional roles in skeletal muscle and cardiovascular system. Mech. Dev. 95:259-265.

15. Gaussin, V., et al. 2003. Common genomic response in different mouse models of beta-adrenergic-induced cardiomyopathy. Circulation. 108:2926-2933.

16. Hwang, D.M., et al.1997. A genome-based resource for molecular cardiovascular medicine: toward a compendium of cardiovascular genes. Circulation. 96:4146-4203.

17. Hwang, D.M., Dempsey, A.A., Lee, C.Y., and Liew, C.C. 2000. Identification of differentially expressed genes in cardiac hypertrophy by analysis of expressed sequence tags. Genomics. 66:1-14.
18. Lim, D.S., Roberts, R., and Marian, A.J. 2001. Expression profiling of cardiac genes in human hypertrophic cardiomyopathy: insight into the pathogenesis of phenotypes. J. Am. Coll. Cardiol. 38:1175-1180.

19. Barki-Harrington, L., Perrino, C., and Rockman, H.A. 2004. Network integration of the adrenergic system in cardiac hypertrophy. Cardiovasc. Res. 63:391-402.

20. Dorn, G.W., II, and Brown, J.H. 1999. Gq signaling in cardiac adaptation and maladaptation. Trends Cardiovasc. Med. 9:26-34.

21. Wettschureck, N., et al. 2001. Absence of pressure overload induced myocardial hypertrophy after conditional inactivation of Galphaq/Galpha11 in cardiomyocytes. Nat. Med. 7:1236-1240.

22. D’Angelo, D.D., et al. 1997. Transgenic Galphaq overexpression induces cardiac contractile failure in mice. Proc. Natl. Acad. Sci. U. S. A. 94:8121-8126.

23. Purcell, N.H., et al. 2004. Extracellular signal-regulated kinase 2 interacts with and is negatively regulated by the LIM-only protein FHL2 in cardiomyocytes. Mol. Cell. Biol. 24:1081-1095.

24. Lange, S., et al. 2002. Subcellular targeting of metabolic enzymes to titin in heart muscle may be mediated by DRAL/FHL-2. J. Cell Sci. 115:4925-4936.

25. Linke, W.A., et al. 1999. I-band titin in cardiac muscle is a three-element molecular spring and is critical for maintaining thin filament structure. J. Cell Biol. 146:631-644.

26. Gille, H., Strahl, T., and Shaw, P.E. 1995. Activation of ternary complex factor Elk-1 by stress-activated protein kinases. Curr. Biol. 5:1191-1200.

27. Yasukawa, H., et al. 2001. Suppressor of cytokine signaling-3 is a biomechanical stress-inducible gene that suppresses gp130-mediated cardiac myocyte hypertrophy and survival pathways. J. Clin. Invest. 108:1459-1467.

28. Esposito, G., Rapacciuolo, A., Naga Prasad, S.V., and Rockman, H.A. 2002. Cardiac hypertrophy: role of G protein-coupled receptors. J. Card. Fail. 8:S409-S414.

29. Chu, P.H., Bardwell, W.M., Gu, Y., Ross, J., Jr., and Chen, J. 2000. FHL2 (SLIM3) is not essential for cardiac development and function. Mol. Cell. Biol. 20:7460-7462.

30. LeWinter, M.M., Wu, Y., Labeit, S., and Granzier, H. 2007. Cardiac titin: structure, functions and role in disease. Clin. Chim. Acta. 375:1-9.

31. Radke, M.H., et al. 2007. Targeted deletion of titin $\mathrm{N} 2 \mathrm{~B}$ region leads to diastolic dysfunction and cardiac atrophy. Proc. Natl. Acad. Sci. U. S. A. 104:3444-3449.

32. Lorenzen-Schmidt, I., et al. 2005. Young MLP deficient mice show diastolic dysfunction before the onset of dilated cardiomyopathy. J. Mol. Cell. Cardiol. 39:241-250.

33. Esposito, G., et al. 2001. Cardiac overexpression of a $G(q)$ inhibitor blocks induction of extracellular signal-regulated kinase and c-Jun $\mathrm{NH}(2)$-terminal kinase activity in in vivo pressure overload. Circulation. 103:1453-1458.

34. Vondriska, T.M., Pass, J.M., and Ping, P. 2004. Scaffold proteins and assembly of multiprotein signaling complexes. J. Mol. Cell. Cardiol. 37:391-397.

35. Kolch, W. 2005. Coordinating ERK/MAPK signal- ing through scaffolds and inhibitors. Nat. Rev. Mol. Cell Biol. 6:827-838.

36. Clapéron, A., and Therrien, M. 2007. KSR and CNK: two scaffolds regulating RAS-mediated RAF activation. Oncogene. 26:3143-3158.

37. Morrison, D.K., and Davis, R.J. 2003. Regulation of MAP kinases signaling modules by scaffold proteins in mammals. Annu. Rev. Cell Dev. Biol. 19:91-118.

38. Sacks, D.B. 2006. The role of scaffold proteins in MEK/ERK signaling. Biochem. Soc. Trans. 34:833-836.

39. Bueno, O.F., and Molkentin, J.D. 2002. Involvement of extracellular signal-regulated kinases $1 / 2$ in cardiac hypertrophy and cell death. Circ. Res. 91:776-781.

40. Bueno, O.F., et al. 2000. The MEK1-ERK1/2 signaling pathway promotes compensated cardiac hypertrophy in transgenic mice. EMBOJ. 19:6341-6350.

41. Harris, I.S., et al. 2004. Raf-1 kinase is required for cardiac hypertrophy and cardiomyocyte survival in response to pressure overload. Circulation. 110:718-723.

42. Purcell, N.H., et al. 2007. Genetic inhibition of cardiac ERK1/2 promotes stress-induced apoptosis and heart failure but has no effect on hypertrophy in vivo. Proc. Natl. Acad. Sci. U. S. A. 104:14074-14079.

43. Wu, Y., Cazorla, O., Labeit, D., Labeit, S., and Granzier, H. 2000. Changes in titin and collagen underlie diastolic stiffness diversity of cardiac muscle. J. Mol. Cell. Cardiol. 32:2151-2162.

44. Trombitas, K., Freiburg, A., Centner, T., Labeit, S., and Granzier, H. 1999. Molecular dissection of N2B cardiac titin's extensibility. Biophys. J. 77:3189-3196.

45. Matsumoto, Y., et al. 2005. Functional analysis of titin/connectin N2-B mutations found in cardiomyopathy. J. Muscle Res. Cell Motil. 26:367-374.

46. Rockman, H.A., et al. 1991. Segregation of atrialspecific and inducible expression of an atrial natriuretic factor transgene in and in vivo murine model of cardiac hypertrophy. Proc. Natl. Acad. Sci. U. S. A. 88:8277-8281.

47. Jones, W.K., et al. 1996. Ablation of the murine alpha myosin heavy chain gene leads to dosage effects and functional deficits in the heart. J. Clin. Invest. 98:1906-1917.

48. Tanaka, N., et al. 1996. Transthoracic echocardiography in models of cardiac disease in the mouse. Circulation. 94:1109-1117.

49. Adams, J.W., et al. 1998. Enhanced Galphaq signaling: a common pathway mediates cardiac hypertrophy and apoptotic heart failure. Proc. Natl. Acad. Sci. U. S. A. 95:10140-10145.

50. Stenmark, H., Vitale, G., Ullrich, O., and Zerial, M. 1995. Rabaptin-5 is a direct effector of the small GTPase Rab5 in endocytic membrane fusion. Cell. 83:423-432.

51. Vojtek, A.B., Hollenberg, S.M., and Cooper, J.A. 1993. Mammalian Ras interacts directly with the serine/threonine kinase Raf. Cell. 74:205-214.

52. Labeit, S., and Kolmerer, B. 1995. Titins: giant proteins in charge of muscle ultrastructure and elasticity. Science. 270:293-296.

53. Sheikh, F., et al. 2006. alpha-E-catenin inactivation disrupts the cardiomyocyte adherens junction, resulting in cardiomyopathy and susceptibility to wall rupture. Circulation. 114:1046-1055. 